\title{
Environmental Noise Classification for Context-Aware Applications
}

\author{
Ling Ma, Dan Smith and Ben Milner \\ School of Computing Sciences, University of East Anglia \\ Norwich, NR4 7TJ, UK \\ \{ling.ma, dan.smith, b.milner\}@uea.ac.uk
}

\begin{abstract}
Context-awareness is essential to the development of adaptive information systems. Much work has been done on developing technologies and systems that are aware of absolute location in space and time; other aspects of context have been relatively neglected. We describe our approach to automatically sensing and recognising environmental noise as a contextual cue for context-aware applications. Environmental noise provides much valuable information about a user's current context. This paper describes an approach to classifying the noise context in the typical environments of our daily life, such as the office, car and city street. In this paper we present our hidden Markov model based noise classifier. We describe the architecture of our system, the experimental results, and discuss the open issues in environmental noise classification for mobile computing.
\end{abstract}

\section{Introduction}

Traditional computer systems run with explicit input and output, such as mouse clicks, keyboard presses and on-screen displays. Users must provide explicit information to the computer and the computer only can give explicit results. Nowadays, mobile computing is increasingly important and people want to access information anytime and anywhere. To exploit this mobility requires systems that can sense appropriate context information and adapt their operation automatically without involving direct user interaction. This information may include location, time, weather, co-located objects, noise, recent events, etc., although many definitions of context have been proposed [10]. Context information is especially important in the mobile computing area where the context and the user's needs change rapidly. We define time, location/co-location and discourse (including environmental noise) as three dimensions of context in mobile computing area of principal interest.

The challenge for context-aware computing is the complexity of capturing, representing, processing, adapting and recording of context information. In a contextaware application, contexts can be captured via different sensors; user feedback can confirm context and be used to improve the model; services can be provided according to the current context and user preferences; finally, context information can be tagged for later retrieval [16]. The notion of context-aware computing was proposed about a decade ago. The Active Badge system [24] - probably the first context-aware application - was published in 1992 and Schilit [22] first introduced the term "context-aware" in 1994. Subsequent work has concentrated on time, identity and location. 
Environmental noise can be a rich source of information about the current situation. For example, we often infer the situation of a respondent in a mobile phone conversation by identifying the background noise and adjusting our response accordingly. The noise classification system described here classifies the audio context in the typical environments of our daily life, such as the office, bar, and city street, to identify the scenes in order to predict the user's needs and adjust the mode of operation accordingly. This work is part of a larger investigation into the integration of multiple sources of context information in a unified framework.

This paper describes the overall design of the context system and is focused on the development and evaluation of an environmental noise classifier. It is organised as follows. In section 2, we review related work in the area of mobile context-aware applications and audio scene classification. Section 3 describes our approach to building an environmental noise classification system, and the methodology to classify auditory scenes into predefined classes. Section 4 presents the experimental results and discussion. In section 5, we describe the overall architecture of the application system. Conclusions and future work are discussed in section 6 .

\section{Related Work}

Most existing context-aware applications are location-based, augmented with timestamp information. The sensors mainly used are short range IR and RF signals, and GPS. Those applications include services, e.g. "Conference assistant" [8], "Office Assistant" [25], or guides, such as "Cyberguide" [1], "Shopping Jacket" [20], and memory aids, e.g. "Stick-e notes" [4], "Memoclip" [2] and "CybreMinder" [9]. Recent surveys [10] [5] [15] show that most existing context-aware applications use identity, time, and location, which are all important and are can be obtained using current technology.

Couvreur [7] has introduced three classifiers (statistical classifiers, adaptable and adaptive classifiers, hidden Markov model based classifiers) to be used in separated noise event recognition (car, truck, airplane etc.) and the attempt to develop a classifier for multiple simultaneous signals. Statistical classifiers have the major drawback of their sensitivity to variations in utilization conditions. Adaptable and adaptive classifiers that adapt to changes in spectra can solve this problem, but do not take the advantage of the time evolving structure of the spectra. Hidden Markov model based classifiers provide a dynamic solution. Gaunard et al. [12] have implemented a HMM-based classifier to recognise five noise events (car, truck, moped, aircraft, train). They observed that the frame length in noise recognition is larger than in speech recognition. Their best results are from a five-state HMM using LPC-cepstral features, which they report gives better results than human listeners. Much work has been done on separating speech from background noise, following [3]; we are interested in recognising everyday scenes (office, car, etc.) rather than sound events.

The above classifiers recognise individual sound events. Only a few classification systems have been proposed to recognise auditory scenes. Peltonen et al. [17] demonstrated that mel-frequency cepstral coefficients out-performed other feature representations. Sawhney [21] classified five everyday noise types, comparing several approaches, of which filterbank with Nearest Neighbour (NN) clearly outperformed the others. Their results indicate that frequency bands generated from filterbank 
analysis of frame-by-frame audio windows provide robust features. Other work (e.g. [6], [11]) has been directed toward recognising both the scene and sound subjects in it, focusing on exhaustively identifying sound events and their relationships in a continuous classifier.

Our approach differs from previous work with its emphasis on rapid, lightweight classification for mobile applications and an adaptive short-duration sampling strategy.

\section{HMM-based Noise Classification}

This section describes a hidden Markov model (HMM) framework for classifying a range of different environmental noises. Classification is based on combining digital signal processing (DSP) technology with pattern recognition methods that have been central to progress in automatic speech recognition [14] over the last 20 years. However there are subtle difference between recognising speech and identifying environmental noises. For example speech is produced from a single source (the human vocal system) which is well modelled, has limitations on the character of sound it can produce and is also constrained to a single location in an environment. Environmental noise, however, has none of these constraints and is a complex sound made up of a mixture of different events. There is no constraint on what these sounds can be and they may emanate from many different localities in the environment. For example consider an office environment; a stationary component may come from an air conditioning fan, a quasi-stationary component from keyclicks, and non-stationary events from people moving around, opening doors and talking.

In this paper, we are interested only in modelling slow-changing attributes of the environmental noise in the audio signal. This means the focus is on recognising the context, or environment, instead of analysing and interpreting discrete sound events.

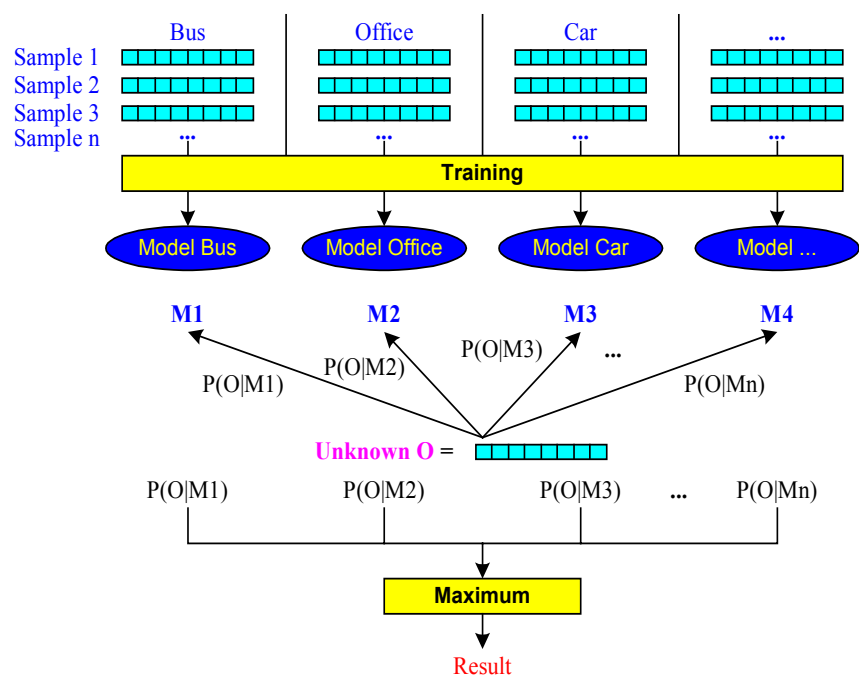

Figure 1. Overall process for feature extraction and classification of noise data 
This section describes four phases which have been used to construct a set of environmental noise models suitable for classification. These are environmental noise database capture via portable recording devices, pre-processing (digitisation, segmentation and labelling), feature exaction using mel-frequency cepstral coefficients (MFCCs) and finally training and testing a set of hidden Markov models (HMMs). Figure 1 shows the overall process for feature extraction and classification of the noise data. These experiments have been performed using the HTK (Hidden Markov Model Toolkit) developed at the Speech, Vision and Robotics Group of the Cambridge University Engineering Department (CUED) [23].

\subsection{Data collection}

A high quality microphone and two portable recording devices (Sony MD and Sony micro cassette) were used to capture the auditory contexts from different scenes. The recordings were designed to cover a range of everyday environments from where users would be accessing information services from mobile devices. The recordings took place in and around the University of East Anglia during the spring and summer of 2002. All data were then labelled manually. Based on the length and quality of the recordings 11 different noise contexts were chosen for initial classification experiments. These are shown in table 1.

\begin{tabular}{|l|l|}
\hline \multicolumn{1}{|c|}{ Scene } & \multicolumn{1}{c|}{ Location } \\
\hline Office & Wolfson Lab at UEA \\
\hline Bus & Across a range of buses \\
\hline Football match & Football match at Norwich City \\
\hline Bar & Graduate student bar in UEA \\
\hline Beach & Great Yarmouth beach \\
\hline Railway station & Norwich railway station \\
\hline Car & Small car in urban driving \\
\hline Laundrette & Laundrette at UEA \\
\hline Street & Norwich city centre on a Saturday \\
\hline Lecture & UEA \\
\hline Silence & -- \\
\hline
\end{tabular}

Table 1. Description of the scenes

\subsection{Data pre-processing and analysis}

Following data collection the audio data was digitised and divided into short duration segments. Digitisation was achieved by sampling the analogue data at $22.050 \mathrm{kHz}$ using 16-bit quantisation. The recordings were then segmented into 3 second duration audio files. This duration was chosen as it is likely to be the length of noise data from which the system would operate in practical applications. Associated with each of these 3 second audio files is a manually created label file which contains information regarding the scene.

Pre-processing resulted in a set of 80 examples for each scene. Of these, 60 examples were used for training and 20 for testing. Therefore, for the 11 scenes 
(including silence), 880 noise examples were used for the initial experiments (660 examples for training and further 220 for testing).

For example, figure 2 shows the spectrogram of the one of the office noise samples. The spectrogram shows time along the abscissa and frequency along the ordinate. Darker regions show more energy present at a particular point in timefrequency.

The spectrogram of the office noise displays a number of distinctive characteristics. For example the vertical lines result from the impulsive noise of keyboard clicks - a feature very characteristic of an office environment. The continuous horizontal line comes from the air conditioning fan which provides another important cue to underlying environment. Other noises also displayed similar identifiable characteristics.

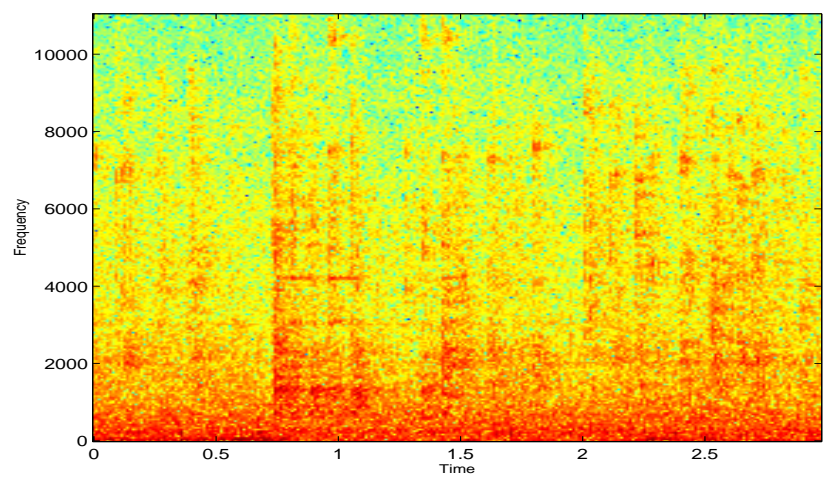

Figure 2. Spectrogram of office noise

\subsection{Feature Extraction}

The purpose of feature extraction is to extract useful discriminative information from the time-domain waveform which will result in a compact set of feature vectors. Researchers have experimented with many different types of feature for use in speech recognition [7][14][17]. The most popular feature used for speech recognition is currently Mel-Frequency Cepstral Coefficients (MFCCs). Peltonen [17] implemented a system for recognising 17 sound events using 11 features individually and obtained the best result with the MFCC features.

MFCC feature extraction begins by estimating the magnitude spectrum of a short duration frame of speech (conventionally using a Fast Fourier Transform, FFT). The magnitude spectrum is then non-linearly quantised using a mel-scale filterbank which models the psychoacoustic properties of the human ear. From this a logarithm is taken and then a discrete cosine transform (DCT) applied [23],

$$
c_{i}=\sqrt{\frac{2}{N}} \sum_{j=1}^{N} m_{j} \cos \left(\frac{\pi i}{N}(j-0.5)\right)
$$

where $c_{i}$ is the $i^{\text {th }}$ MFCC, $N$ is the number of filterbank channels and $m_{j}$ the output of the $j^{\text {th }}$ mel-scale filterbank channel. This results in the MFCC vector. 
For the experiments used in this work the speech was first pre-emphasised and then a Hamming window used to extract $25 \mathrm{~ms}$ duration frames of audio. These frames were extracted every $10 \mathrm{~ms}$. A 23 -channel mel filterbank was applied to the resultant magnitude spectrum. Following the DCT, truncation resulted in a 12-D MFCC vector. This was augmented by a log energy term to give a 13-D static feature vector. To improve performance both the velocity and acceleration were computed and this resulted in a 39-D feature vector which was used for training and testing.

\subsection{HMM Training and Testing}

For modelling the different environmental noises a set of HMMs has been created. These provide a powerful statistical method of dynamically characterizing the observed noise samples in time and frequency. The HMM has also been very successful in automatic speech recognition applications [19].

A HMM consists of a series of stationary states which are connected together by a Markov chain. This allows the time-varying nature of signals to be modelled as a progression through a series of stationary states. This makes the HMM an appropriate model for representing the non-stationary behaviour of environmental noises. More detailed HMM theory is given in [19]. For noise modelling a left-to-right HMM topology was used (figure 3). This model topology has a self-transition and a left-toright transition to the next state. The self-transition is used to model contiguous features in the same state. The left-to-right transition enables a progression when the next quasi-stationary segment evolves. In such a topology, each state has a statedependent output probability distribution that is used to interpret the observable noise signal. Progression through the states is described by a transition probability matrix.

For the left-to-right HMM, an important parameter to determine is the number of states. To a certain extent the choice of model topology depends on the amount of available training data and the signal to be modelled. For the time-varying nature of environment noise, more states are generally required and this also depends on the duration of the signal. For our three second noise signal, we have implemented from 3 to 21 state HMMs.

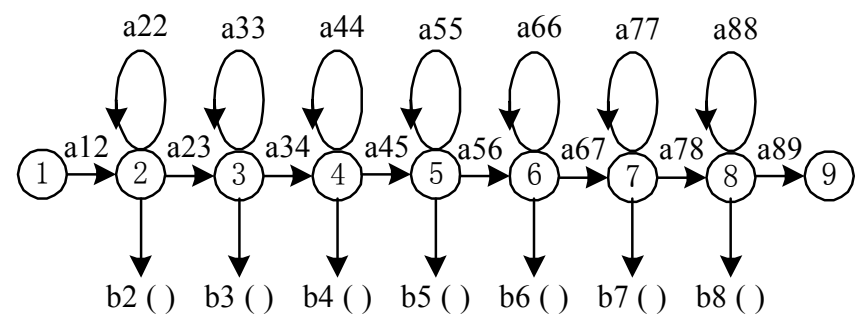

Figure 3. A nine-state left-to-right HMM model topology

\section{Experimental Results}

The HMMs were trained using the 60 noise examples from each of the 11 different noise scenes to give a set of $11 \mathrm{HMMs}$. A preliminary test was performed to identify the optimal number of states for the models. Ten different numbers of states 
were tested; 3, 5, 7, 9, 11, 13, 15, 17, 19 and 21 states. The classification performance for each number of states in the HMM is shown in figure 4.

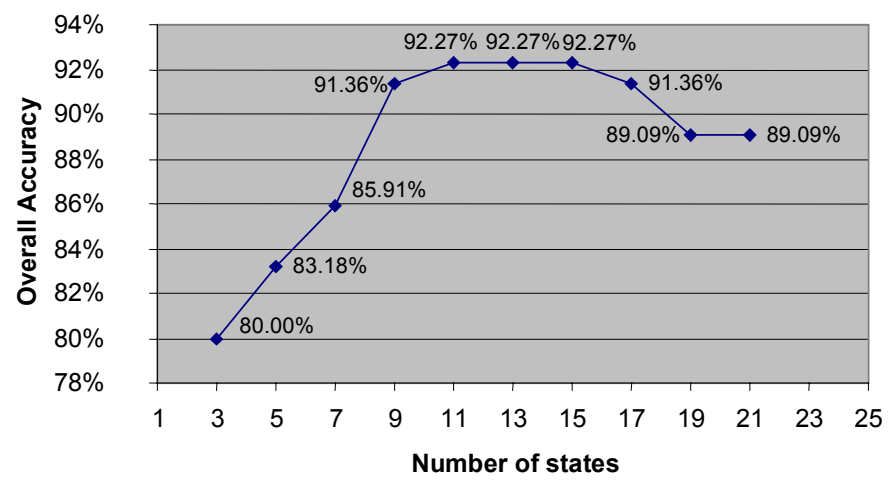

Figure 4. Overall accuracy from 3 to 21 states

From the figure we see that accuracy of the 3 -state HMM is $80.00 \%$ and as the number of states increases the accuracy is improved up to a maximum of $92.27 \%$ at 11,13 and 15 states. At higher numbers of states the accuracy begins to reduce. This indicates that the number of states in the HMM has a significant impact on the overall classification accuracy. In general, more states in the HMM does allow better modelling of the underlying noise characteristics. On the other hand, a model with more states will have more parameters which will requires more training data to estimate accurately. In addition, the duration of our samples is 3 seconds and too many states in the HMM may cause overfitting. Therefore the limited amount of training data and relatively short duration of the samples cause the accuracy to decrease with a number of states higher than 15 .

Using the result from the 11-state HMM topology, the accuracy of the individual scenes is shown in table 2 . These ranged from $75 \%$ to $100 \%$, with the office, football match, beach, laundrette and silence giving 100\% classification accuracy for the 20 examples tested of each. Worst performance was obtained for identifying street noise. This attained only $75 \%$ accuracy but can be attributed to the fact the this is probably the most diverse noise type.

The accuracy of noise recognition depends on a number of factors such as the amount and coverage of the training data, the feature extraction component, the allowable computational complexity, and the model parameters. The noise classifier designed in this system is not capable of recognising multiple and simultaneously occurring environmental noises. For example sitting in an office with a car is passing by would cause a conflict. Similar problems also occur when identifying similar noise scenes. 


\begin{tabular}{|c|c|c|c|c|c|c|c|c|c|c|c|}
\hline \multicolumn{12}{|c|}{ Result and Confusion matrix for 11 scenes } \\
\hline Accuracy $\%$ & $\stackrel{n}{\tilde{n}}$ & 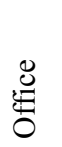 & 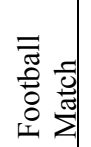 & 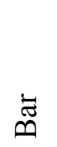 & $\begin{array}{l}\frac{\tilde{D}}{\tilde{D}} \\
\tilde{D}\end{array}$ & تే & 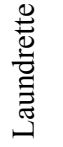 & 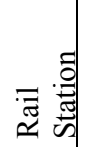 & 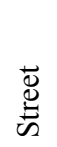 & 苞 & $\begin{array}{l}0 \\
\frac{0}{0} \\
\frac{0}{\pi}\end{array}$ \\
\hline Bus & 95 & 0 & 5 & 0 & 0 & 0 & 0 & 0 & 0 & 0 & 0 \\
\hline Office & 0 & 100 & 0 & 0 & 0 & 0 & 0 & 0 & 0 & 0 & 0 \\
\hline Football Match & 0 & 0 & 100 & 0 & 0 & 0 & 0 & 0 & 0 & 0 & 0 \\
\hline Bar & 15 & 0 & 0 & 85 & 0 & 0 & 0 & 0 & 0 & 0 & 0 \\
\hline Beach & 0 & 0 & 0 & 0 & 100 & 0 & 0 & 0 & 0 & 0 & 0 \\
\hline Car & 0 & 0 & 0 & 0 & 10 & 85 & 0 & 0 & 5 & 0 & 0 \\
\hline Laundrette & 0 & 0 & 0 & 0 & 0 & 0 & 100 & 0 & 0 & 0 & 0 \\
\hline Rail Station & 0 & 0 & 0 & 0 & 0 & 0 & 0 & 90 & 10 & 0 & 0 \\
\hline Street & 0 & 0 & 0 & 0 & 0 & 0 & 10 & 15 & 75 & 0 & 0 \\
\hline Lecture & 0 & 0 & 0 & 0 & 0 & 0 & 0 & 0 & 15 & 85 & 0 \\
\hline Silence & 0 & 0 & 0 & 0 & 0 & 0 & 0 & 0 & 0 & 0 & 100 \\
\hline \multicolumn{12}{|c|}{ Overall accuracy: $92.27 \%$} \\
\hline
\end{tabular}

Table 2. Noise classification results and confusion matrix

For simultaneous classification and to get improved discrimination when classifying similar scenes, we may consider how humans solve these sorts of classification problems. Peltonen's [18] human listening test shows that humans distinguish similar scenes by catching pieces of sound events in them (e.g. music, clinking glasses and conversation in a bar). Previous research has successfully modelled distinct sound events [12] [17]. The short sampling strategy we use does not require a dedicated audio channel and can obtain noise samples during periods of speech inactivity. A dedicated noise channel would facilitate alternative recognition strategies.

\section{Context System Design}

The basic context-aware application framework has a client-server architecture, shown in figure 5 . The server side comprises a set of noise models, a database of new samples, a noise classification application and a web application server. The current client application, mostly written in Java, consists of an audio recorder, noise recogniser, noise analyser, context logger, browser and update connection. These are lightweight processes which can easily run on limited capacity devices. The audio recorder periodically samples the environmental noise ( 3 seconds per minute by default). The noise recogniser classifies the noise. The noise analyser reads the result file and initiated any actions required. The context logger writes each captured context to an XML record. The browser is used as a memory aid, by recalling context details. The update connection updates the HMM definitions.

The current system senses two contexts, environmental noise and time. The microphone is a sensor to sense the environmental noise. The built-in clock is the time sensor. The time is automatically called updated every time a new noise context is captured. If a changed or uncertain context is detected, samples are taken more 
frequently until the change is confirmed or the context is clarified. The values read from sensors periodically are continually fed to an extensible log in XML format. The state of the system and a number of sampling parameters can be controlled by the user. The $\log$ can be used for later analysis to refine the classifier's performance and to detect longer duration events.

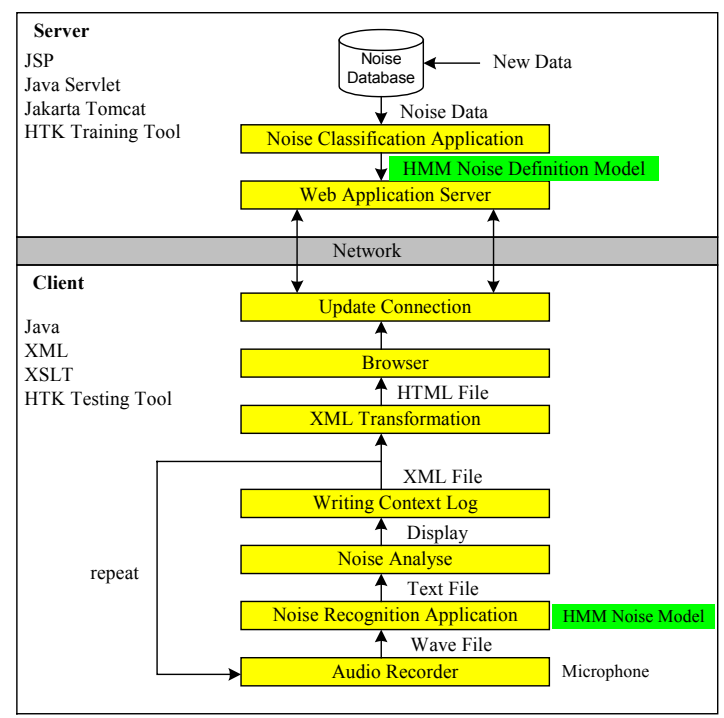

Figure 5. System architecture

\section{Conclusions and Future Work}

We have described our HMM-based environmental noise classifier which we trained on a database of manually gathered samples. The overall recognition accuracy of 11 typical scenes in our daily life (including silence) is $92.27 \%$. The recognition accuracy of individual scenes ranged from $75 \%$ to $100 \%$. We intend undertaking further experiments, using more samples and more scenes from different locations, to compare the result of using different features, classification methods and algorithms.

We have described our initial client-server context-aware application. The current version of this system is able to capture, recognise and track environmental noise changes as a user changes locations. We are developing this system to provide: a range of customisable output responses and modalities appropriate to the user's situation, extending the range of input modalities, and to increasing the range of context data.

\section{References}

[1] Abowd G. D., Atkeson C. G., Hong J., Long S., Kooper R., and Pinkerton M., Cyberguide: A mobile context-aware tour guide, ACM Wireless Networks, 1997.

[2] Beigl M. MemoClip: A Location based Remembrance Appliance, Personal Technologies, 4(4): 230-233, September 2000. 
[3] Brown G.J. and Cooke M. P., Computational Auditory Scene Analysis. Computer Speech and Language, 8, pp. 297-336, 1994.

[4] Brown P. J., STICK-E NOTES: changing notes and contexts -- the SeSensor module and the loading of notes, EP-odd, January 1996.

[5] Chen G., Kotz D., A Survey of Context-Aware Mobile Computing, Research Dept. of Computer Science, Dartmouth College, 2000.

[6] Clarkson B., Sawhney N. and Pentland A., Auditory Context Awareness via Wearable Computing, Proc. of the 1998 Workshop on Perceptual User Interfaces (PUI'98), San Francisco, CA, USA, November 1998.

[7] Couvreur C., Environmental Sound Recognition: A Statistical Approach, PhD thesis, Faculte Polytechnique de Mons, Belgium, June 1997.

[8] Dey A. K., Salber D., Abowd G. D. and Futakawa M., The Conference Assistant: Combining Context-Awareness with Wearable Computing, The Third International Symposium on Wearable computers, 1999.

[9] Dey A. K. and Abowd G. D., CybreMinder: A Context-Aware System for Supporting reminders, In Proceeding of the Second International Symposium on Handheld and Ubiquitous Computing, HUC 2000.

[10] Dey A.K., Abowd G. D., Towards a Better Understanding of Context and ContextAwareness, CHI 2000 Workshop on the What, Who, Where, When, and How of ContextAwareness, 2000.

[11] Ellis D., Prediction-Driven Computational auditory Scene Analysis For Dense Sound Mixtures, the ESCA workshop on the Auditory Basis of Speech Perception, Keele UK, July 1996.

[12] Gaunard P., Mubikangiey C. G., Couvreur C. and Fontaine V., Automatic Classification Of Environmental Noise Events By Hidden Markov Models, Applied Acoustics, 1998.

[13] Harter A., Hopper A., Steggles P., Ward A. and Webster P., The Anatomy of a ContextAware Application, ACM/IEEE Mobile Computing and Networking, 2002.

[14] Huang X., Acero A. and Hon H., Spoken Language Processing, Prentice Hall, 2001.

[15] MIT media Lab, http://cac.media.mit.edu:8080/contextweb/jsp/projects.jsp

[16] Pascoe, J., The Stick-e Note Architecture: Extending the Interface Beyond the User, International Conference on Intelligent User Interfaces, Orlando, Florida, USA. ACM. pp. 261-264, 1997.

[17] Peltonen V., Tuomi J., Klapuri A., Huopaniemi J. and Sorsa T., Computational Auditory Scene Recognition. In Proc. International Conference on Acoustic, Speech and Signal Processing, Orlando, Florida, May 2002.

[18] Peltonen V.T.K., Eronen A.J., Parviainen M. P. and Klapuri A.P., Recognition of Everyday Auditory Scenes: Potentials, Latencies and Cues, $110^{\text {th }}$ Convention Audio Engineering Society, 2001.

[19] Rabiner L. R., A tutorial on hidden Markov models and selected application in speech recognition, Proc. IEEE, vol. 77, no. 2, pp. 257-286, Feb. 1989.

[20] Randell C., Muller H., The Shopping Jacket: Wearable Computing for the Consumer, Personal Technologies vol.4 no.4.

[21] Sawhney N., Situational Awareness from Environmental Sounds, 1997.

[22] Schilit B., Adams N., Want R., Context-Aware Computing Applications, IEEE Workshop on Mobile Computing Systems and Applications, 1994.

[23] The HTK Book Version 3.1, Cambridge University Engineering Department, December 2001, http://htk.eng.cam.ac.uk.

[24] Want R., Hopper A., Falcao V., Gibbons J., The Active Badge Location System, ACM Transactions on Information Systems, 10(1) 1992.

[25] Yan H. and Selker T., Context-aware office assistant, In Proceedings of the 2000 International Conference on Intelligent User Interfaces, New Orleans, LA, January 2000. 